\title{
Association of secondary displacement of distal radius fractures with cortical bone quality at the distal radius
}

\author{
A. M. Daniels ${ }^{1,2}$ - H. M. J. Janzing ${ }^{1} \cdot$ C. E. Wyers ${ }^{2,3,4} \cdot$ B. van Rietbergen ${ }^{5,6} \cdot$ L. Vranken ${ }^{2,3} \cdot$ R. Y. Van der Velde ${ }^{2,3}$. \\ P. P. M. M. Geusens ${ }^{4,7} \cdot$ S. Kaarsemaker ${ }^{8} \cdot$ M. Poeze ${ }^{2,9} \cdot$ J. P. Van den Bergh ${ }^{2,3,4,7}$
}

Received: 18 March 2020 / Accepted: 15 October 2020 / Published online: 31 October 2020

(c) The Author(s) 2020

\begin{abstract}
Introduction The aim of this study was to investigate the associations of patient characteristics, bone mineral density (BMD), bone microarchitecture and calculated bone strength with secondary displacement of a DRF based on radiographic alignment parameters.

Materials and methods Dorsal angulation, radial inclination and ulnar variance were assessed on conventional radiographs of a cohort of 251 patients, 38 men and 213 women, to determine the anatomic position of the DRF at presentation (primary position) and during follow-up.

Secondary fracture displacement was assessed in the non-operatively treated patients $(N=154)$ with an acceptable position, preceded $(N=97)$ or not preceded $(N=57)$ by primary reduction (baseline position). Additionally, bone microarchitecture and calculated bone strength at the contralateral distal radius and tibia were assessed by HR-pQCT in a subset of, respectively, 63 and 71 patients.

Outcome Characteristics of patients with and without secondary fracture displacement did not differ. In the model with adjustment for primary reduction [OR 22.00 (2.27-212.86), $p=0.008$ ], total [OR 0.16 (95\% CI 0.04-0.68), $p=0.013$ ] and cortical [OR 0.19 (95\% CI 0.05-0.80], $p=0.024$ ] volumetric BMD (vBMD) and cortical thickness [OR 0.13 (95\% CI $0.02-0.74), p=0.021]$ at the distal radius were associated with secondary DRF displacement. No associations were found for other patient characteristics, such as age gender, BMD or prevalent vertebral fractures.

Conclusions In conclusion, our study indicates that besides primary reduction, cortical bone quality may be important for the risk of secondary displacement of DRFs.
\end{abstract}

Keywords Distal radius fracture (DRF) · Fracture displacement · High-resolution peripheral quantitative CT (HR-pQCT) · Bone microarchitecture and strength $\cdot$ Primary reduction

Electronic supplementary material The online version of this article (https://doi.org/10.1007/s00402-020-03658-2) contains supplementary material, which is available to authorized users.

\section{A. M. Daniels}

adaniels@viecuri.nl

1 Department of Surgery, VieCuri Medical Centre, Tegelseweg 210, 5912 BL Venlo, The Netherlands

2 NUTRIM School for Nutrition and Translational Research in Metabolism, Maastricht University, Universiteitssingel 40, 6229 ER Maastricht, The Netherlands

3 Department of Internal Medicine, Subdivision of Endocrinology, VieCuri Medical Centre, Tegelseweg 210, 5912 BL Venlo, The Netherlands

4 Department of Internal Medicine, Maastricht University Medical Centre, P. Debyelaan 25, 6229 HX Maastricht, The Netherlands
5 Orthopaedic Biomechanics, Department of Biomedical Engineering, Eindhoven University of Technology, De Rondom 70, 5612 AP Eindhoven, The Netherlands

6 Department of Orthopaedic Surgery, Research School CAPHRI, Maastricht University, Universiteitssingel 40, 6229 ER Maastricht, The Netherlands

7 Faculty of Medicine, Hasselt University, Martelarenlaan 42, 3500 Hasselt, Belgium

8 Department of Orthopaedic Surgery, VieCuri Medical Centre, Tegelseweg 210, 5912 BL Venlo, The Netherlands

9 Department of Surgery, Subdivision of Traumatology, Maastricht University Medical Centre, P. Debyelaan 25, 6229 HX Maastricht, The Netherlands 


\section{Introduction}

Standard initial management for distal radius fractures (DRFs) at the emergency department (ED) is cast immobilisation (CI) preceded by closed reduction in the case of a dislocated fracture. Further management depends on the anatomic position, DRFs with acceptable position can be managed non-operatively [1]. Dislocated intra-articular DRFs often require surgical fixation to restore and retain correct fracture position. Patient characteristics, such as age and comorbidities, must be taken into account when making this decision [2-4]. It is of great importance to identify fractures at risk for displacement to achieve the most adequate treatment. By being able to anticipate earlystage instability, unnecessary manipulation can be prevented, surgical treatment options can be discussed timely and a reduction in complications such as mal-union might be accomplished.

In 1989, five risk factors for DRF instability were identified by Lafontaine et al. namely primary dorsal angulation exceeding 20 degrees, dorsal comminution, involvement of the radio-carpal joint, styloid ulnae fracture and patients aged over 60 years [5]. From that time on, many prediction rules for instability have been developed, some previous risk factors could not be confirmed by new studies and new risk factors for secondary displacement, such as radial shortening, have been identified [6-11]. In this debate, little attention was paid to the influence of bone properties such as bone mineral density (BMD), bone microarchitecture and calculated bone strength. BMD can be assessed using bone densitometry, whereas bone microarchitecture and separate assessment of trabecular and cortical bone requires spatial resolution of less than $200 \mu \mathrm{m}$. Recently, a non-invasive method for the assessment of bone microarchitecture at the distal radius and tibia using high-resolution peripheral quantitative CT (HR-pQCT) has become available.

The aim of this study was to investigate the associations of patient characteristics, BMD [measured by dual-energy X-ray absorptiometry (DXA) and High-Resolution peripheral quantitative CT (HR-pQCT)], bone microarchitecture and calculated bone strength with secondary displacement of a DRF based on radiographic alignment parameters.

\section{Methods}

\section{Study population}

This cohort comprised patients aged 50-90 years presenting with a radiologically confirmed DRF, between
November 2013 and June 2016. All consecutive patients were referred to the Fracture Liaison Service (FLS) and included in this study if they attended the FLS. After exclusion of patients with high-energy trauma (as this study focuses on fall-related fractures), osteomyelitis and bone metastasis, 251 patients with a recent DRF were included in this cross-sectional cohort study.

At the FLS, patients received a detailed evaluation according to the Dutch guideline for treatment of osteoporosis. The evaluation consisted of a questionnaire assessing risk factors for falls, fracture risk, medical history including medication use, and daily dietary calcium intake. Additionally, blood samples were collected to identify metabolic disorders and a DXA measurement with vertebral fractures assessment (VFA) was performed 3-4 months after trauma (Fig. 1). If indicated, anti-osteoporosis treatment or treatment of newly diagnosed metabolic bone disorders was initiated according to current guidelines [12].

Of the 251 patients with a DRF included in this study, 71 participated in an observational 3-year follow-up study at the FLS ("Prospective evaluation of bone strength, physical activity, falls, subsequent fractures and mortality in patients presenting with a recent clinical fracture"). Approval was obtained from an institutional Review Board prior to performing the study (METC NL 45707.072.13). In that study, patients consented with HR-pQCT measurements of the distal radius $(N=63)$ and tibia $(N=71)$ and the baseline data are used for the HR-pQCT part of the current study. HR-pQCT measurements were conducted approximately 3 weeks after the DXA scan was performed (Fig. 1).

\section{DRF position and classification}

All conventional radiographs (antero-posterior and lateral) were used for assessment of alignment parameters, comprising angulation $($ dorsal $=\mathrm{DA}$, volar $/ \mathrm{palmar}=\mathrm{VA})$, radial inclination (RI) and ulnar variance (UV). DA/VA is measured on the lateral radiograph and represents the angle between a line perpendicular to the longitudinal axis of the radius and a line along the articular surface of the distal radius. RI is measured on the antero-posterior radiograph and represents the angle between a line connecting the tip of the radial styloid and the most ulnar point of the distal radius and a second line perpendicular to the longitudinal axis of the radius. UV represents the length of the ulna compared to the radius. According to the Dutch Guideline, DRFs were classified as fractures with an 'unacceptable position' when at least one of the following criteria was met; DA $>15^{\circ}$, $\mathrm{VA}>20^{\circ}, \mathrm{RI} \leq 15^{\circ}$ and $\mathrm{UV}>5 \mathrm{~mm}$ (Fig. 2a-c) [13]. Position at presentation, referred to as primary position, was assessed on the first radiographs of every patient. If reduction was applied the position was reassessed on radiographs following reduction, this position is referred to as baseline 


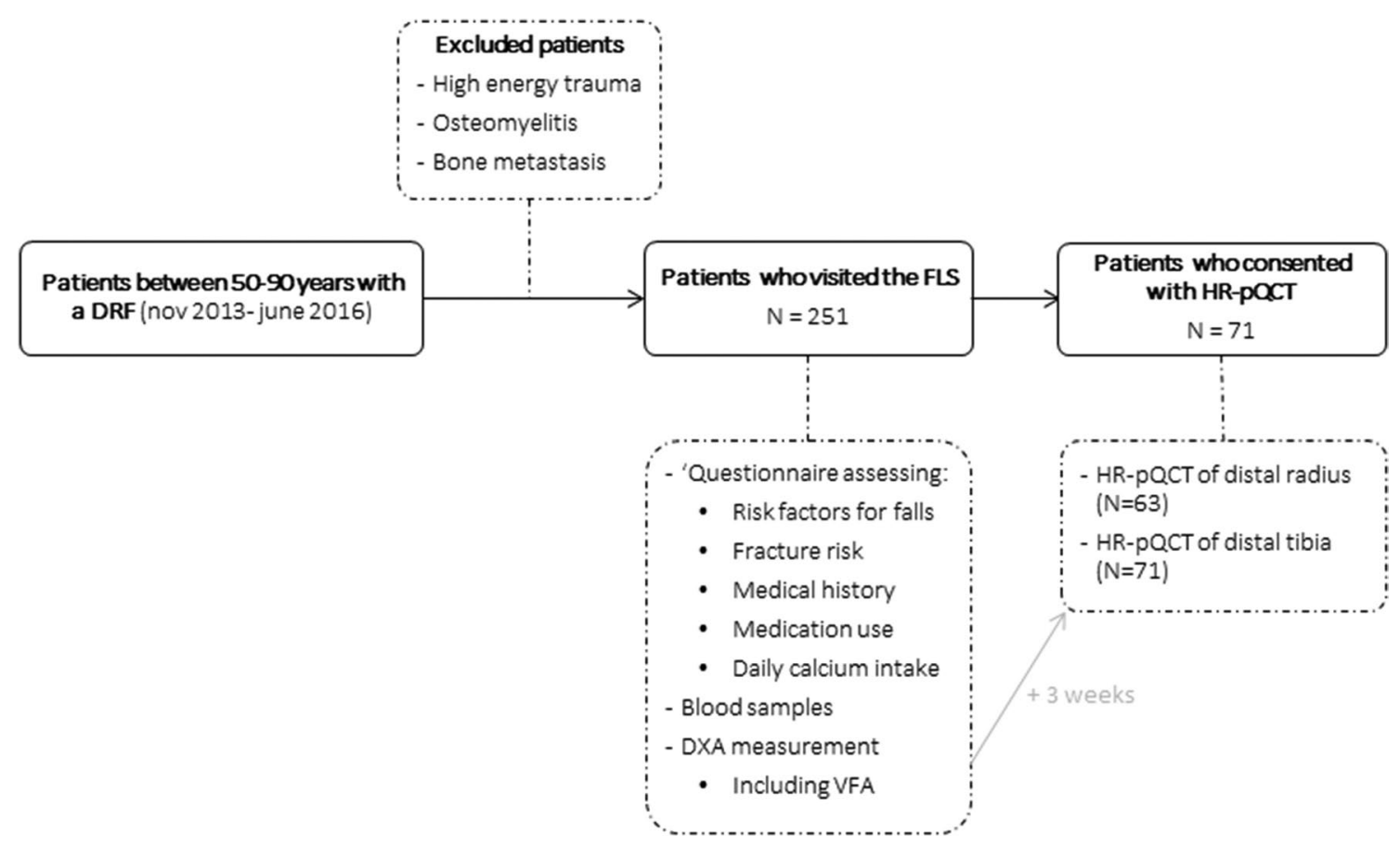

Fig. 1 Flowchart of patients included in this study and corresponding investigations
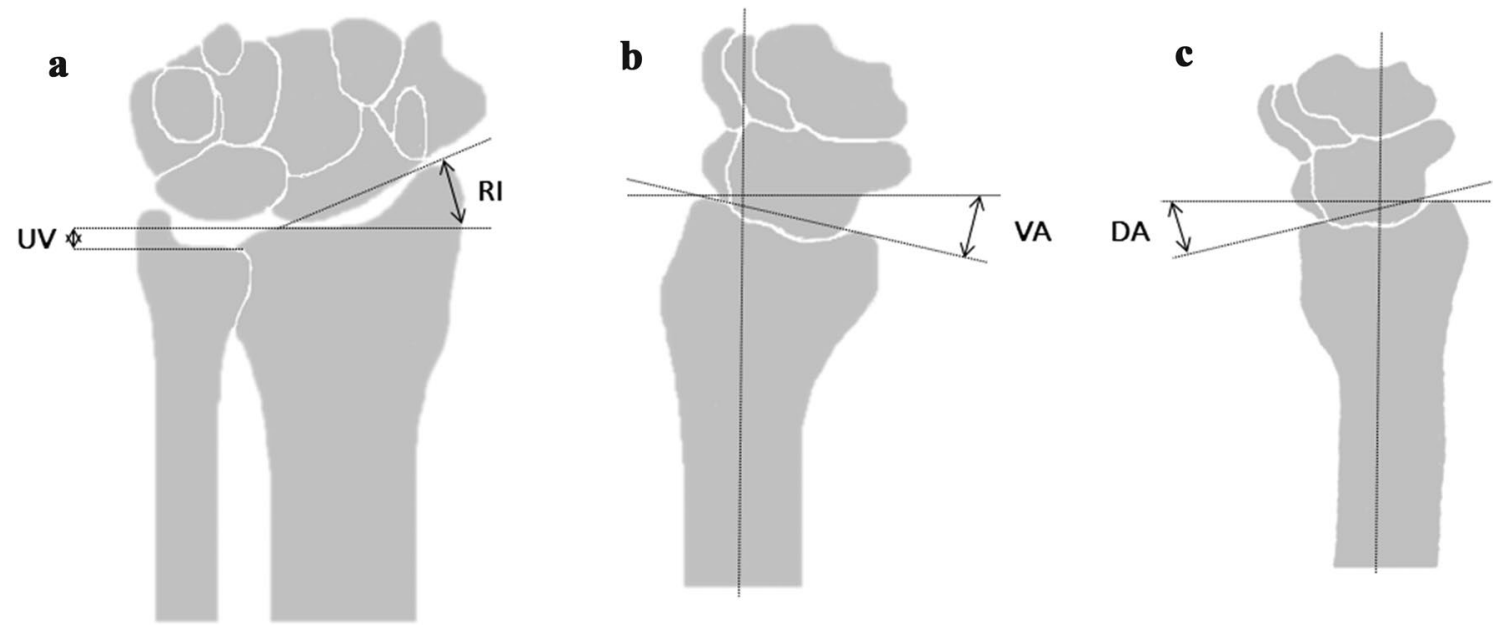

Fig. 2 a Radiographic alignment parameters (RI/UV) to assess the distal radius [48]. b Radiographic alignment parameters (VA) to assess the distal radius [48]. c Radiographic alignment parameters (DA) to assess the distal radius [48]

position. Adequate reduction was defined as regaining an acceptable position according to the criteria. In unreduced fractures with repeated radiographs immediately after cast immobilisation, baseline position is the position as measured on the repeated radiographs. In unreduced fractures without repeated radiographs baseline position was equivalent to primary position. All subsequent radiographs were assessed individually and used for secondary fracture displacement assessment, starting at baseline position. Patients with primary surgical intervention, an unacceptable baseline position or without follow-up radiographs were excluded from secondary fracture displacement assessment (Fig. 3). Secondary fracture displacement was defined as a displacement of the DRF that resulted in an unacceptable position, after an adequate baseline position in non-operatively treated patients.

All fractures were classified based on the AO/OTA classification on baseline plain radiographs by two independent investigators. DRFs were classified into three main types, namely type A (extra-articular), type B (partial articular) and 


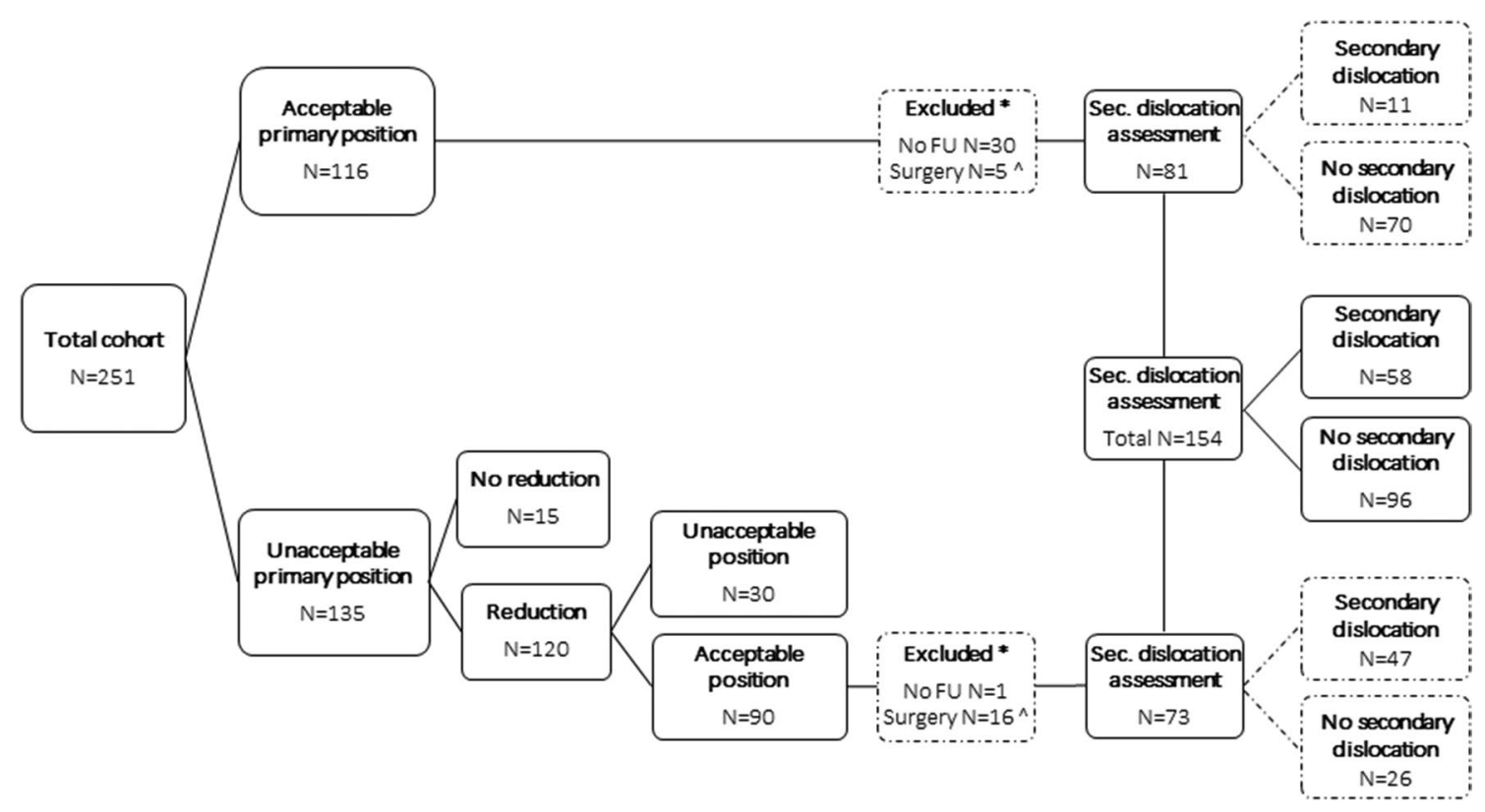

Fig. 3 Flowchart of patient distribution and (eligibility for) secondary fracture displacement assessment

type C (complete articular). Assessment by a third independent investigator was necessary for 51 patients with discrepant initial classification and resulted in agreement on another 47 fractures. For the remaining four fractures, conformity was reached by all three investigators in a consensus meeting.

\section{DXA and VFA}

Two-dimensional BMD was measured at the lumbar spine (LS; L1-L4), total hip (TH) and femoral neck (FN) using DXA (Hologic QDR 4500, Hologic Inc., Bedford, MA, USA). Areal BMD measurements $\left(\mathrm{g} / \mathrm{cm}^{2}\right)$, were categorized according to the WHO criteria based on the lowest $T$-score at the LS, TH or FN into normal BMD ( $T$-score $\geq-1)$, osteopenia $(T$-score between -1 and -2.5$)$ and osteoporosis $(T$-score $\leq-2.5)[14]$.

VFA was performed on the DXA lateral spine images using quantitative morphometric assessment of vertebral height. The method described by Genant et al. was used to classify the severity of vertebral fractures (VFs); grade 1 (mild fracture, with vertebral height loss of 20-25\%), grade 2 (moderate fracture with height loss of $25-40 \%$ ) and grade 3 (severe fracture with height loss $>40 \%$ ) [15].

\section{HR-pQCT}

The second-generation HR-pQCT (XtremeCT II; Scanco Medical AG, Brüttisellen, Switzerland) was used to scan the contralateral radius and ipsilateral tibia. Scans were conducted and evaluated according to the standard protocol of the manufacturer (effective energy of $68 \mathrm{kVp}$, tube current of $1470 \mu \mathrm{A}$ and $43 \mathrm{~ms}$ integration time) $[16,17]$. The reference line was placed on the joint surface of the distal radius and tibia. The area to be scanned starts $9.0 \mathrm{~mm}$ proximally to the reference line and ends $1.2 \mathrm{~mm}$ distally to the reference line. Motion-induced degradation of the images was graded according to the method of Pialat et al. [17]. Images were processed according to the manufacturer's standard protocol. The following parameters were analyzed: total, trabecular and cortical bone area $\left[\mathrm{cm}^{2}\right]$, volumetric bone mineral density (vBMD) for the total, trabecular and cortical compartment $\left[\mathrm{mgHA} / \mathrm{cm}^{3}\right]$, trabecular bone volume fraction, trabecular number $\left[\mathrm{mm}^{-1}\right]$, trabecular thickness [mm], trabecular separation [mm], cortical thickness [mm], cortical perimeter [mm], cortical porosity [\%] and cortical pore diameter $[\mathrm{mm}]$. In addition, micro-finite element analyses (micro-FEA) were generated by directly converting bone voxels in the segmented image to brick elements [18, 19]. Elements were assigned a Young's modulus of $10 \mathrm{GPa}$ and a Poisson's ratio of 0.3 and for each model, four tests were simulated [20]. The first load case represented a 'high friction' compression test with a prescribed displacement in the axial direction of $1 \%$ of the total length, from which the compression stiffness $[\mathrm{kN} / \mathrm{mm}]$ as well as the estimated strength was calculated [21-23]. The second load case represented a prescribed rotation of $0.01 \mathrm{rad}$ around the longitudinal axis from which the torsional stiffness $[\mathrm{kNmm} / \mathrm{rad}]$ was calculated. A third and fourth load case represented a prescribed rotation of $0.01 \mathrm{rad}$ applied around the sagittal and transversal axes, respectively, thus inducing a state of pure bending in two directions, from which the bending stiffness in each direction was calculated. These four load cases 
were included to test if the fracture type is associated with a reduced stiffness in a specific loading direction.

\section{Statistical analysis}

Data were analyzed using IBM SPSS Statistics, version 24 (IBM Corporation 1989, 2016). Normal distribution was tested with Q-Q plots and Kolmogorov-Smirnov analysis. Depending on the distribution, data are presented as mean with standard deviation (SD) or median with interquartile range (IQR). Chi-square tests were used to analyze differences in patient characteristics between the groups. Independent samples $t$ tests were used to compare HRpQCT parameters between patients with an acceptable and unacceptable primary position and between patients with and without secondary fracture displacement. Logistic regression analysis was used to investigate the independent association between secondary fracture displacement (yes vs. no) and HR-pQCT parameters. Bivariate analyses were conducted for primary reduction and all standardized scores ( $z$-scores) of the HR-pQCT parameters for both the HR-pQCT radius group $(N=30)$ and HR-pQCT tibia group $(N=36)$. The cutoff value for significance to assess parameters in a multivariable model was defined as $p \leq 0.10$. Due to the small sample size, multivariable analyses with adjustment for age and primary reduction were conducted separately for each significant HR-pQCT parameter in bivariate analysis. Receiver operating characteristic (ROC) analyses with area under the curve (AUC) measurements were conducted for all significant variables in the HR-pQCT analyses. Adjustment for age was conducted because of the potential effect on bone microarchitecture and strength. Significance level was set as $\alpha=0.05$.

\section{Results}

\section{Primary position}

Of 251 patients, 38 men (15\%) and 213 women (85\%) with a mean age of 67 years $(\mathrm{SD} \pm 9), 116(46 \%)$ had an acceptable and 135 (54\%) an unacceptable primary position. One patients had a fracture with 20 degrees volar angulation and was therefore subjected to surgery. None of the fractures with volar angulation exceeded the range of 20 degrees. Patients with a DRF with primary unacceptable position were significantly older (69 vs. 66 years, $p=0.015$ ) and had a lower body weight (68.6 vs. $73.5 \mathrm{~kg}, p=0.035)$ and BMI $\left(25.5\right.$ vs. $\left.27.7 \mathrm{~kg} / \mathrm{m}^{2}, p=0.048\right)$ than DRF patients with acceptable position [Table $\mathrm{S}-1$ ]. The proportion of $\mathrm{AO} /$ OTA type $\mathrm{A}$ and $\mathrm{B}$ fractures was higher and type $\mathrm{C}$ fractures was lower in patients with an acceptable primary position compared to patients with an unacceptable primary position $(p<0.001)$. There was no difference in gender, BMD, number and severity of VFs, alcohol intake, smoking, calcium intake and vitamin D levels. Neither was there a difference in bone microarchitecture and strength measured by HRpQCT between patients with a DRF with primary acceptable (tibia $N=31$, radius $N=26$ ) and unacceptable position (tibia $N=40$, radius $N=35$ ) [data not shown]. HR-pQCT scans of the distal tibia were conducted in all patients $(N=71)$, while distal radius scans could not be conducted in eight of these patients due to the presence of a bilateral DRF at the time of the study or a prior DRF at the contralateral side. Furthermore, two patients with unacceptable primary position had a bad-quality HR-pQCT of the distal radius and were therefore not included in HR-pQCT analysis.

\section{Secondary fracture displacement}

Reduction was conducted in 120/135 patients with a primary unacceptable position with a success rate of $75 \%$. This resulted in 206 patients with an acceptable baseline position. In 20 patients, surgery was conducted because of comminution of the fracture or patients' preference. One patient had a fracture with 20 degrees volar angulation and, therefore, underwent surgery. After exclusion of these 21 patients and 31 patients without follow-up, 154 were eligible and assessed for secondary fracture displacement. Median follow-up with radiographs was 35 days (interquartile range 45 days), with numerous follow-up visits in this time range (Fig. 3). Secondary displacement occurred within the first 2 weeks in 39 patients (67\%), in the third week in 12 patients (21\%) and seven patients (12\%) had a DRF displacement after 1 month. When comparing patients with secondary fracture displacement $(N=58)$ to those without $(N=96)$, we found no differences for age, gender distribution, BMI, BMD, number and severity of VFs, smoking, alcohol intake, calcium intake and vitamin D levels (Table 1). Primary reduction was significantly associated with secondary DRF displacement [OR 12.53 (95\% CI 4.60-34.09), $p<0.001$ ] in 154 patients.

HR-pQCT tibia and radius scans were available in 36 (23\%), respectively, 30 (20\%) patients for evaluation of secondary fracture displacement. Characteristics of patients with HR-pQCT measurement $(N=36)$ were not different compared to those without HR-pQCT $(N=118)$. Neither was there a difference in the proportion of secondary vs. non secondary dislocated fractures (Table 2).

At the distal radius (measured in 30 patients), total [OR 0.27 (95\% CI 0.10-0.73), $p=0.010$ ] and cortical [OR 0.31 (95\% CI 0.12-0.80), $p=0.016]$ vBMD and cortical thickness [0.32 (95\% CI 0.13-0.80), $p=0.015$ ] were significantly lower in patients with secondary dislocated fractures. There were no differences for trabecular parameters and micro-FEA. The strongest determinant for secondary 
Table 1 Characteristics of 154 patients with and without secondary fracture dislocation

\begin{tabular}{|c|c|c|c|}
\hline & $\begin{array}{l}\text { No secondary } \\
\text { dislocation } \\
N=96\end{array}$ & $\begin{array}{l}\text { Secondary } \\
\text { dislocation } \\
N=58\end{array}$ & $p$-value \\
\hline Female & $83(87)$ & $54(93)$ & 0.202 \\
\hline Age (year)* & $66[14]$ & $68.5[13]$ & 0.054 \\
\hline Weight $(\mathrm{kg})^{*}$ & $73.3[22.8]$ & $66.6[22.0]$ & 0.101 \\
\hline Height (m) & $1.63 \pm 0.08$ & $1.62 \pm 0.06$ & 0.340 \\
\hline $\operatorname{BMI}\left(\mathrm{kg} / \mathrm{m}^{2}\right)^{*}$ & $26.4[6.9]$ & $25.4[7.4]$ & 0.174 \\
\hline \multicolumn{4}{|l|}{$\mathrm{AO}$} \\
\hline A & $57(59.4)$ & $36(62.1)$ & \multirow[t]{3}{*}{0.334} \\
\hline $\mathrm{B}$ & $14(14.6)$ & $4(6.9)$ & \\
\hline $\mathrm{C}$ & $25(26.0)$ & $18(31.0)$ & \\
\hline \multicolumn{4}{|l|}{ BMI category } \\
\hline$<30$ (non obees) & $63(72.4)$ & $40(76.9)$ & \multirow[t]{2}{*}{0.557} \\
\hline$\geq 30$ (obees) & $24(27.6)$ & $12(23.1)$ & \\
\hline \multicolumn{4}{|l|}{ Bone densitometry } \\
\hline Normal BMD & $16(16.7)$ & $8(13.8)$ & \multirow[t]{3}{*}{0.710} \\
\hline Osteopenia & $48(50.0)$ & $27(46.6)$ & \\
\hline Osteoporosis & $32(33.4)$ & $23(39.7)$ & \\
\hline \multicolumn{4}{|l|}{ VFA } \\
\hline No VF & 87 (90.6) & $51(87.9)$ & \multirow[t]{2}{*}{0.595} \\
\hline$\geq 1$ Grade $2 / 3 \mathrm{VF}$ & $9(9.4)$ & $7(12.1)$ & \\
\hline \multicolumn{4}{|l|}{ Smoking } \\
\hline Never & $40(42.6)$ & $23(41.1)$ & \multirow[t]{3}{*}{0.689} \\
\hline Past smoker & $42(44.7)$ & $23(41.1)$ & \\
\hline Current smoker & $12(12.8)$ & $10(17.9)$ & \\
\hline \multicolumn{4}{|l|}{ Alcohol intake } \\
\hline$<1$ unit/day & $28(30.4)$ & $21(39.6)$ & \multirow[t]{2}{*}{0.260} \\
\hline$\geq 1$ unit/day & $64(69.6)$ & $32(60.4)$ & \\
\hline $\begin{array}{l}\text { Calcium intake (mg/ } \\
\text { day)* }\end{array}$ & 797 [396] & $843[428]$ & 0.968 \\
\hline \multicolumn{4}{|c|}{ 25-OH Vitamin D (nmol/l) } \\
\hline$<30$ (deficiency) & $8(8.3)$ & $9(15.5)$ & \multirow[t]{3}{*}{0.308} \\
\hline 30-50 (insufficiency) & $32(33.3)$ & $15(25.9)$ & \\
\hline$>50$ (sufficiency) & $56(58.3)$ & $34(58.6)$ & \\
\hline
\end{tabular}

$B M I$ body mass index, $B M D$ bone mineral density, $V F$ vertebral fracture

Data missing: length (14), weight (14), calcium intake (4), alcohol use (9), smoking (4)

Normally distributed data are presented as mean (SD)

Non normally distributed data $*$ are presented as median [IQR]

fracture displacement was primary reduction [OR 22.00 (95\% CI 2.27-212.86), $p=0.008$ ]. After adjustment for primary reduction, total $[0.16$ (95\% CI $0.04-0.68)$, $p=0.013]$ and cortical [0.19 (95\% CI 0.05-0.80), $p=0.024]$ vBMD and cortical thickness [0.13 (95\% CI $0.02-0.74), p=0.021]$ were significantly associated with secondary fracture displacement. Adjustment for age did not change the association of HR-pQCT parameters with secondary displacement of a DRF [Table S-2].

At the distal tibia (measured in 36 patients), total vBMD [OR 0.35 (95\% CI 0.13-0.92), $p=0.034$ ] and cortical pore diameter [OR 0.37 (95\% CI $0.14-0.94), p=0.036$ ] at the distal tibia were significantly lower in patients with secondary dislocated fractures, while there were no differences for trabecular parameters and micro-FEA. After adjustment for primary reduction, both total vBMD [OR $0.06(95 \% \mathrm{CI}$ $0.01-0.64), p=0.020$ ] and cortical thickness [OR 0.22 (95\% CI $0.06-0.84), p=0.027]$ were significantly associated with secondary fracture displacement. After adjustment for age, none of the HR-pQCT parameters at the distal tibia was significantly associated with secondary displacement of a DRF [Table S-3].

\section{Discussion}

DRFs with an unacceptable position are generally reduced at the ED as the first step of treatment. Recovery of alignment is thought to be important to preserve adequate function [1, 24]. Our study showed that fractures with an unacceptable position at need for reduction are, after adequate reduction, at high risk for secondary displacement. In addition, this study shows for the first time, that secondary fracture displacement was independently associated with lower total and cortical vBMD and lower cortical thickness at the distal radius, measured by HR-pQCT, after adjustment for primary reduction while no other clinical parameter including BMD, vertebral fractures was associated with secondary fracture displacement. The HR-pQCT results are clinically relevant as the odds of secondary fracture displacement is $81-87 \%$ higher in patients with lower total and cortical vBMD and cortical thickness at the distal radius.

Previous data regarding timing of displacement are controversial. Some studies reported that all DRFs dislocate in the first two weeks, whereas others describe it as a gradual process $[6,25,26]$. Our data indicate that $88 \%$ of all displacements occur in the first 3 weeks. Although nearly significant $(p=0.054)$, our data did not confirm previous findings that secondary fracture displacement was associated with age [5-8]. This might be due to the age limit of 50-90 years in our study, whereas Abbaszadegan et al. included patients from the age of 18 years. Gender, BMI, VFA, smoking/alcohol status, calcium intake and vitamin D levels were not associated with secondary displacement of a DRF in our study.

The association between BMD, measured by DXA, and the ability to maintain adequate position of a DRF has previously been investigated. Clayton et al. (2009) concluded that osteoporosis (T-score $<-2.5$ ) was associated with secondary DRF displacement in a cohort of 137 patients aged over 
Table 2 Characteristics of patients assessed for secondary dislocation with and without HR-pQCT measurement $(N=154)$

\begin{tabular}{|c|c|c|c|}
\hline & With HR-pQCT $N=36$ & $\begin{array}{l}\text { Without HR-pQCT } \\
N=118\end{array}$ & $p$-value \\
\hline Secondary dislocation & $12(33)$ & $24(24)$ & 0.540 \\
\hline Female & $32(89)$ & $105(89)$ & 0.597 \\
\hline Age (year)* & $68[12]$ & $67[16]$ & 0.673 \\
\hline Weight $(\mathrm{kg})^{*}$ & $71.5[21]$ & $69.3[21]$ & 0.981 \\
\hline Height (m) & $1.65 \pm 0.06$ & $1.63 \pm 0.07$ & 0.118 \\
\hline BMI* & $25.4[5.0]$ & $26.4[8.4]$ & 0.476 \\
\hline \multicolumn{4}{|l|}{$\mathrm{AO}$} \\
\hline A & $24(66.7)$ & $69(58.5)$ & \multirow[t]{3}{*}{0.648} \\
\hline $\mathrm{B}$ & $4(11.1)$ & $14(11.9)$ & \\
\hline $\mathrm{C}$ & $8(22.2)$ & 35 (29.7) & \\
\hline \multicolumn{4}{|l|}{ BMI category } \\
\hline$<30$ (non obees) & $27(81.8)$ & $76(71.7)$ & \multirow[t]{2}{*}{0.247} \\
\hline$\geq 30$ (obees) & $6(18.2)$ & $30(28.3)$ & \\
\hline \multicolumn{4}{|l|}{ Bone densitometry } \\
\hline Normal BMD & $6(16.7)$ & $18(15.3)$ & \multirow[t]{3}{*}{0.939} \\
\hline Osteopenia & $18(50.0)$ & $57(48.3)$ & \\
\hline Osteoporosis & $12(33.3)$ & $43(36.3)$ & \\
\hline \multicolumn{4}{|l|}{ VFA } \\
\hline No VF & $31(86.1)$ & 107 (90.7) & \multirow[t]{2}{*}{0.532} \\
\hline$\geq 1$ Grade $2 / 3 \mathrm{VF}$ & $5(13.9)$ & $11(9.3)$ & \\
\hline \multicolumn{4}{|l|}{ Smoking } \\
\hline Never & $14(38.9)$ & $49(43.0)$ & \multirow[t]{3}{*}{0.863} \\
\hline Past smoker & $17(47.2)$ & $48(42.1)$ & \\
\hline Current smoker & $5(13.9)$ & $17(14.9)$ & \\
\hline \multicolumn{4}{|l|}{ Alcohol use } \\
\hline$<1$ unit/day & $8(22.2)$ & $41(37.6)$ & \multirow[t]{2}{*}{0.090} \\
\hline$\geq 1$ unit/day & $28(77.8)$ & $68(62.4)$ & \\
\hline Calcium intake (mg/day)* & 855 [379] & $780[404]$ & 0.205 \\
\hline \multicolumn{4}{|l|}{ 25-OH Vitamin D (nmol/l) } \\
\hline$<30$ (deficiency) & $2(5.6)$ & $15(12.7)$ & \multirow[t]{3}{*}{0.256} \\
\hline 30-50 (insufficiency) & $9(25.0)$ & $38(32.2)$ & \\
\hline$>50$ (sufficiency) & $25(69.4)$ & $65(55.1)$ & \\
\hline
\end{tabular}

$B M I$ body mass index, $B M D$ bone mineral density, $V F$ vertebral fracture

Data missing: length (14), weight (14), calcium intake (4), alcohol use (9), smoking (4)

Normally distributed data are presented as mean (SD). Non normally distributed data * as median [IQR]
55 [27]. On the contrary, Robin et al. (2014) studied patients aged over 65 years with a displaced DRF and concluded that there was no relationship between BMD, measured by DXA, and the ability to maintain position after adequate reduction [28]. These findings are in line with our study where we observed no difference in or association with BMD, measured by DXA, between patients with and without secondary fracture displacement.

Although cortical integrity is widely suggested to play a role in DRF stability $[5,6,29,30]$, assessment of cortical comminution is frequently conducted without a clear definition $[5,9]$. For example, dorsal comminution is assessed on conventional radiographs, but exact measurement is not possible due to limited resolution. A non-invasive method recently available for the assessment of bone microarchitecture at the extremities is HR-pQCT [23, 31, 32]. In our study, secondary fracture displacement was independently associated with lower total and cortical vBMD and lower cortical thickness at the distal radius, measured by HR-pQCT. At the distal tibia, lower total vBMD and lower cortical thickness appeared to be determinants for secondary DRF displacement, however, after adjustment for age, these HR-pQCT parameters were no longer associated with secondary DRF displacement. Although the HR-pQCT measurements were performed in a limited number of patients we found significant associations with total and cortical vBMD and cortical 
thickness at the distal radius. We believe that the comparison of the affected with the unaffected distal radius is the most appropriate way to study the associations of skeletal parameters with secondary fracture displacement. To best of our knowledge, this is the first study demonstrating the association of bone microarchitecture with secondary DRF displacement.

Some issues were limiting in our study. First, closed reduction was performed by the treating physician at the ED by which some variability might exist in the decision, the technique and quality (as result of experience level) of reduction. Second, due to the retrospective design of our study, radiographic alignment parameters were only assessed at the fractured radius. Since both wrists of one individual can be considered as symmetrical [33], van Eerten et al. [34] assessed the implementation of a technique comparing the fractured site with the unaffected side. They conclude that only reproducibility of radial inclination measurement, and not of radial length or dorsal/volar angulation, improved after implementation of the new template technique. Third, fracture assessment in this study was conducted using radiographs since previous literature shows no significant increase of inter and intra observer agreement of the AO classification of DRFs when using CT [35]. However, recent studies suggests that additional CT scanning may be of importance for the accuracy of scoring the fracture types [36, 37]. Fourth, for classification of the complexity of DRFs, the AO/OTA classification was used. This is one of many available classification systems being the Frykman, Fernandez, Melone and universal classification system [35, 38, 39], unfortunately none of these systems has perfect reproducibility rates [35, 40-42]. In contrast to other classification systems, the AO/OTA classification has a strong intra- and inter-observer reliability for assessment of the main type (A—extra articular, B-partially articular, $\mathrm{C}-$ complete articular). Classifying the AO subtype is not recommended based on the poor intra- and inter-observer reliability [43]. In concordance with this, consensus rate in our study for two independent investigators was $79.7 \%$. Furthermore, classification of main type was in line with previous published papers [44, 45]. Fourth, since assessment of BMD and VFs was only possible in FLS attenders, we studied a selected cohort of patients presenting at the ED with a DRF. Due to the retrospective design of our study, not all patients underwent HR-pQCT. However, there was no difference between patients with and without HR-pQCT measurement and no difference in secondary fracture displacement distribution between the total cohort and the subgroup with HR-pQCT measurement. Accordingly, the study results of the subset of patients with HR-pQCT are representative for and can be extrapolated to our total cohort of patients with a DRF. Finally, as described in the result section, 14 patients with an unacceptable position were treated non-operatively. This was due to the fact that they were not willing or suitable, as judged by their treating physician, to undergo surgery. It is well founded not to subject older patients with multiple comorbidities to manipulation/surgery since it is proven to be of minimal value $[46,47]$, however, this might have caused bias in our study.

In conclusion, our data demonstrate that the most important determinant for secondary displacement of a DRF was primary reduction. However, while other patient characteristics, BMD and VF status were not associated with secondary fracture displacement, lower total and cortical vBMD and lower cortical thickness at the distal radius were independently associated with secondary displacement of a DRF. This indicates that besides primary reduction, cortical bone quality may be important for the risk of secondary displacement of DRFs.

Author contributions AMD: conceptualization, data curation, analysis, funding acquisition, investigation, methodology, project administration, writing original draft, reviewing/editing. HMJJ: conceptualization, analysis, methodology, project administration reviewing/editing, supervision. CWW: conceptualization, data curation, analysis, methodology. reviewing/editing. BR: conceptualization, methodology, reviewing/ editing. LV: data curation, investigation, reviewing/editing. RYV: data curation, investigation, reviewing/editing. PPMMG: conceptualization, methodology, reviewing/editing. SK: conceptualization, methodology, reviewing/editing. MP: conceptualization, analysis, methodology, project administration reviewing/editing, supervision. JPB: conceptualization, analysis, funding acquisition, methodology, project administration reviewing/editing, supervision.

Funding This work was supported by the VieCuri MC trust for research and innovation, Grant number E17.31.033.4.

\section{Compliance with ethical standards}

Conflict of interest Bert van Rietbergen is a consultant for Scanco Medical AG. All other authors state that they have no conflicts of interest.

Ethical approval Approval was obtained from an institutional Review Board prior to performing the study (METC NL 45707.072.13).

Open Access This article is licensed under a Creative Commons Attribution 4.0 International License, which permits use, sharing, adaptation, distribution and reproduction in any medium or format, as long as you give appropriate credit to the original author(s) and the source, provide a link to the Creative Commons licence, and indicate if changes were made. The images or other third party material in this article are included in the article's Creative Commons licence, unless indicated otherwise in a credit line to the material. If material is not included in the article's Creative Commons licence and your intended use is not permitted by statutory regulation or exceeds the permitted use, you will need to obtain permission directly from the copyright holder. To view a copy of this licence, visit http://creativecommons.org/licenses/by/4.0/. 


\section{References}

1. Mauck BM, Swigler CW (2018) Evidence-based review of distal radius fractures. Orthop Clin North Am 49(2):211-222

2. Orbay JL, Fernandez DL (2004) Volar fixed-angle plate fixation for unstable distal radius fractures in the elderly patient. J Hand Surg Am 29(1):96-102

3. Rozental TD, Blazar PE (2006) Functional outcome and complications after volar plating for dorsally displaced, unstable fractures of the distal radius. J Hand Surg Am 31(3):359-365

4. Diaz-Garcia RJ et al (2011) A systematic review of outcomes and complications of treating unstable distal radius fractures in the elderly. J Hand Surg Am 36(5):824-35.e2

5. Lafontaine M, Hardy D, Delince P (1989) Stability assessment of distal radius fractures. Injury 20(4):208-210

6. Abbaszadegan H, Jonsson U, von Sivers K (1989) Prediction of instability of Colles' fractures. Acta Orthop Scand 60(6):646-650

7. Adolphson P, Abbaszadegan H, Jonsson U (1993) Computerassisted prediction of the instability of Colles' fractures. Int Orthop 17(1):13-15

8. Hove LM et al (1994) Prediction of secondary displacement in Colles' fracture. J Hand Surg Br 19(6):731-736

9. Mackenney PJ, McQueen MM, Elton R (2006) Prediction of instability in distal radial fractures. J Bone Joint Surg Am 88(9):1944-1951

10. Nesbitt KS, Failla JM, Les C (2004) Assessment of instability factors in adult distal radius fractures. J Hand Surg Am 29(6):1128-1138

11. Walenkamp MM et al (2016) Predictors of unstable distal radius fractures: a systematic review and meta-analysis. J Hand Surg Eur 41(5):501-515

12. CBO DIfHI, Richtlijn Osteoporose en Fractuurpreventie, Derde Herziening [Dutch]. Utrecht, Dutch Institute for Healthcare Improvement CBO, The Netherlands, (2011)

13. Heelkunde, $N V v$, Richtlijn distale radius fracturen: diagnostiek en behandeling. Nederlandse Vereniging voor Heelkunde, (2010)

14. Kanis JA et al (2008) A reference standard for the description of osteoporosis. Bone 42(3):467-475

15. Genant HK et al (1993) Vertebral fracture assessment using a semiquantitative technique. J Bone Miner Res 8(9):1137-1148

16. Manske SL et al (2015) Human trabecular bone microarchitecture can be assessed independently of density with second generation HR-pQCT. Bone 79:213-221

17. Pialat JB et al (2012) Visual grading of motion induced image degradation in high resolution peripheral computed tomography: impact of image quality on measures of bone density and microarchitecture. Bone 50(1):111-118

18. Agarwal $\mathrm{S}$ et al (2016) In vivo assessment of bone structure and estimated bone strength by first- and second-generation HRpQCT. Osteoporos Int 27(10):2955-2966

19. van Rietbergen B et al (1995) A new method to determine trabecular bone elastic properties and loading using micromechanical finite-element models. J Biomech 28(1):69-81

20. de Jong JJ et al (2014) Assessment of the healing process in distal radius fractures by high resolution peripheral quantitative computed tomography. Bone 64:65-74

21. Pistoia $\mathrm{W}$ et al (2004) Image-based micro-finite-element modeling for improved distal radius strength diagnosis: moving from bench to bedside. J Clin Densitom 7(2):153-160

22. Mueller TL et al (2011) Computational finite element bone mechanics accurately predicts mechanical competence in the human radius of an elderly population. Bone 48(6):1232-1238

23. Hosseini HS et al (2017) Fast estimation of Colles' fracture load of the distal section of the radius by homogenized finite element analysis based on HR-pQCT. Bone 97:65-75
24. Batra S, Gupta A (2002) The effect of fracture-related factors on the functional outcome at 1 year in distal radius fractures. Injury 33(6):499-502

25. Abbaszadegan H, von Sivers K, Jonsson U (1988) Late displacement of Colles' fractures. Int Orthop 12(3):197-199

26. Altissimi $M$ et al (1994) Early and late displacement of fractures of the distal radius. The prediction of instability. Int Orthop 18(2):61-65

27. Clayton RA et al (2009) Association between decreased bone mineral density and severity of distal radial fractures. J Bone Joint Surg Am 91(3):613-619

28. Robin BN et al (2014) Relationship of bone mineral density of spine and femoral neck to distal radius fracture stability in patients over 65. J Hand Surg Am 39(5):861-6.e3

29. Alemdaroglu KB et al (2010) Three-point index in predicting redisplacement of extra-articular distal radial fractures in adults. Injury 41(2):197-203

30. LaMartina J et al (2015) Predicting alignment after closed reduction and casting of distal radius fractures. J Hand Surg Am 40(5):934-939

31. D'Elia $\mathrm{G}$ et al (2009) Bone fragility and imaging techniques. Clin Cases Miner Bone Metab 6(3):234-246

32. de Jong JJA et al (2016) Fracture repair in the distal radius in postmenopausal women: a follow-up 2 years postfracture using HRpQCT. J Bone Miner Res 31(5):1114-1122

33. Hollevoet $\mathrm{N}$ et al (2000) Comparison of palmar tilt, radial inclination and ulnar variance in left and right wrists. J Hand Surg Br 25(5):431-433

34. van Eerten PV et al (2008) An X-ray template assessment for distal radial fractures. Arch Orthop Trauma Surg 128(2):217-221

35. Arealis G et al (2014) Does the CT improve inter- and intraobserver agreement for the AO, Fernandez and Universal classification systems for distal radius fractures? Injury 45(10):1579-1584

36. Kleinlugtenbelt YV et al (2017) Classification systems for distal radius fractures. Acta Orthop 88(6):681-687

37. Li SL, Wang MY, Lu Y (2017) Diagnostic value of CT scan for AO B3 fracture of distal radius. Beijing Da Xue Xue Bao Yi Xue Ban 49(4):675-679

38. Lill CA et al (2003) Impact of bone density on distal radius fracture patterns and comparison between five different fracture classifications. J Orthop Trauma 17(4):271-278

39. Plant CE et al. (2015) Is it time to revisit the AO classification of fractures of the distal radius? Inter- and intra-observer reliability of the AO classification. Bone Joint J 97-b(6): 818-23

40. Yinjie $Y$ et al (2020) A retrospective evaluation of reliability and reproducibility of Arbeitsgemeinschaftfur Osteosynthesefragen classification and Fernandez classification for distal radius fracture. Medicine (Baltimore) 99(2):e18508

41. Porrino JA Jr et al (2014) Fracture of the distal radius: epidemiology and premanagement radiographic characterization. AJR Am J Roentgenol 203(3):551-559

42. Belloti JC et al (2008) Are distal radius fracture classifications reproducible? Intra and interobserver agreement. Sao Paulo Med J 126(3):180-185

43. Wæver D et al (2018) Distal radius fractures are difficult to classify. Injury 49(Suppl 1):S29-s32

44. Dhainaut A et al (2014) Exploring the relationship between bone density and severity of distal radius fragility fracture in women. J Orthop Surg Res 9:57

45. Jayakumar P et al (2017) AO distal radius fracture classification: global perspective on observer agreement. J Wrist Surg $6(1): 46-53$

46. Beumer A, McQueen MM (2003) Fractures of the distal radius in low-demand elderly patients: closed reduction of no value in 53 of 60 wrists. Acta Orthop Scand 74(1):98-100 
47. Levin LS, Rozell JC, Pulos N (2017) Distal radius fractures in the elderly. J Am Acad Orthop Surg 25(3):179-187

48. Meinberg EG et al (2018) Fracture and dislocation classification compendium-2018. J Orthop Trauma 32(Suppl 1):S1-s170
Publisher's Note Springer Nature remains neutral with regard to jurisdictional claims in published maps and institutional affiliations. 